Louisiana State University

LSU Digital Commons

Faculty Publications

Department of Geology and Geophysics

$1-1-1997$

\title{
Ab initio elasticity of three high-pressure polymorphs of silica
}

Bijaya B. Karki

The University of Edinburgh

Lars Stixrude

Georgia Institute of Technology

Jason Crain

The University of Edinburgh

Follow this and additional works at: https://digitalcommons.Isu.edu/geo_pubs

\section{Recommended Citation}

Karki, B., Stixrude, L., \& Crain, J. (1997). Ab initio elasticity of three high-pressure polymorphs of silica. Geophysical Research Letters, 24 (24), 3269-3272. https://doi.org/10.1029/97GL53196

This Article is brought to you for free and open access by the Department of Geology and Geophysics at LSU Digital Commons. It has been accepted for inclusion in Faculty Publications by an authorized administrator of LSU Digital Commons. For more information, please contact ir@lsu.edu. 


\title{
Ab initio elasticity of three high-pressure polymorphs of silica
}

\author{
Bijaya B. Karki, Lars Stixrude ${ }^{\dagger}$ and Jason Crain \\ Department of Physics and Astronomy, The University of Edinburgh, Edinburgh, EH9 3JZ, Scotland, U.K. \\ †School of Earth and Atmospheric Sciences, Georgia Institute of Technology \\ Atlanta, GA 30332-0340, U.S.A.
}

\begin{abstract}
Full elastic constant tensors of three highpressure polymorphs of silica: stishovite, $\mathrm{CaCl}_{2}$-type and columbite-type ( $\alpha-\mathrm{PbO}_{2}$ structure); are determined at lower mantle pressures from first-principles using the plane wave pseudopotential method within the local density approximation. The calculated zero pressure athermal elastic moduli are within a few percent of the experiments. We find that the elastic properties of silica are strongly pressure dependent. The shear wave velocity decreases rapidly (by $60 \%$ ) and the anisotropy increases rapidly (by a factor of five) between 40 and $47 \mathrm{GPa}$ prior to the transition from stishovite to the $\mathrm{CaCl}_{2}$ structure at $47 \mathrm{GPa}$. At this phase transition, the isotropically averaged shear wave velocity changes discontinuously by $60 \%$, while the S-wave polarization anisotropy decreases by a factor of two. The transformation of the $\mathrm{CaCl}_{2}$ phase to the columbite phase at 98 $\mathrm{GPa}$ is accompanied by a discontinuous change of 1-2 \% in elastic wave velocity and decrease by a factor of two in anisotropy. We suggest that even a small amount of silica in the lower mantle may contribute significantly to observed seismic anisotropy, and may provide an explanation of observed seismic reflectivity near $1000 \mathrm{~km}$.
\end{abstract}

\section{Introduction}

High-pressure elasticity of silica is important for several reasons: First, silica occurs in Earth's crust and portions of subducting slabs in significant amounts; second, it serves as a prototype for high-pressure silicate minerals; and third, an elastic instability occurs in $\mathrm{SiO}_{2}$ resulting in a structural transformation. In addition, the elastic properties of $\mathrm{SiO}_{2}$ are likely to be strongly pressure dependent since it undergoes a series of structural transformations, from $\alpha$-quartz to, coestite, stishovite, $\mathrm{CaCl}_{2}$, columbite $\left(\alpha-\mathrm{PbO}_{2}\right.$ structure) and finally to pyrite, in the pressure range from 0 to 240 GPa [Karki et al., 1997b]. The extrapolations of the zero or low pressure experimental results to high pressures are not straightforward in the case of silica. Therefore, an explicit determination of its elastic properties as a function of pressure is essential.

Starting from stishovite (a rutile-type polymorph of $\mathrm{SiO}_{2}$ ), we have recently predicted three pressure-induced structu-

Copyright 1997 by the American Geophysical Union.

Paper number 97GL53196.

0094-8534/97/97GL-53196\$05.00 ral transformations in silica up to $240 \mathrm{GPa}$ [Karkı et al., $1997 \mathrm{~b}]$. The transition from the stishovite to the orthorhombic $\mathrm{CaCl}_{2}$ phase occurs at $47 \mathrm{GPa}$ in excellent agreement with Raman spectroscopic observations ( $50 \pm 3 \mathrm{GPa}$ ) [Kingma et al., 1995]. The transition from the $\mathrm{CaCl}_{2}$ to the columbite structure occurs at $98 \mathrm{GPa}$ while the subsequent transition from the columbite to the pyrite structure occurs at a pressure (226 GPa) well below the core-mantle boundary. Thus if free silica exists in the lower mantle, it is expected to undergo a series of phase transformations; from stishovite (stable to a depth of approximately $1200 \mathrm{~km}$ ), to $\mathrm{CaCl}_{2}-$ structure (1180-2250 km depth), and finally to columbite (2250-2890 km depth).

The study of high-pressure behaviour of the elastic properties of silica is limited both experimentally and theoretically. No experimental measurements of the elastic constants exist beyond ambient conditions [Weidner et al., 1982]. Using the lineraised augmented plane wave (LAPW) method, Cohen [1991, 1992] has calculated the pressure variation of only a subset of the elastic constants for the stishovite and $\mathrm{CaCl}_{2}$ phase. Here we determine from first-principles the full elastic constant tensors of three high-pressure polymorphs of silica: stishovite, $\mathrm{CaCl}_{2}$ and columbite; study the pressure dependence of the wave velocities and anisotropy and discuss the geophysical implications of our results.

\section{Method}

The computations are based on density functional theory (DFT) within the local density approximation Kohn and Sham [1965]. The optimized, norm-conserving, non-local pseudopotentials are used. A plane wave basis set with a $900 \mathrm{eV}$ cutoff is used to expand the valence wavefunctions at the special k-points generated by a $4 \times 4 \times 4$ mesh [Monkhurst and Pack, 1976, ], and the finite basis set corrections to total energies and stresses are included [Francis and Payne, 1990]. The computational details have been given in the work by Karki et al., [1997b]. The fully optimized structures of the stishovite, $\mathrm{CaCl}_{2}$ - and columbite-type phases of silica [Karki et al., 1997b] are used to derive the elastic properties. The full elastic coefficient tensors are determined from the computation of the stresses generated by small deformations of the unit cell with simultaneous relaxation of the ions in the deformed cell [Wentzcovitch et al., 1995; Karki et al., $1997 a, c]$. Strains of different magnitudes are applied and the values of the elastic moduli are derived from the resulting non-linear stress-strain relations in the appropriate limit of zero strain. 


\section{Results}

\section{Elastic Stiffness Coefficients}

The elastic stiffness coefficients $\left(c_{i j}\right)$, six for the tetragonal phase (stishovite) and nine for the orthorhombic phases $\left(\mathrm{CaCl}_{2}\right.$ and columbite), of silica are determined as function of pressure up to $140 \mathrm{GPa}$ which includes the pressures of stability of the three phases and also exceeds the pressure of Earth's mantle-core boundary. The predicted zero pressure values of the elastic moduli of stishovite are in excellent agreement with experimental data [Weidner et al., 1982] and the LAPW results [Cohen, 1991, 1992] as shown in Table I. There are not yet experimental data with which to compare the pressure dependence of the elastic moduli predicted by our calculations (Fig. 1).

In stishovite, $c_{11}$ increases slowly with pressure up to $40 \mathrm{GPa}$ and then decreases on further compression whereas $c_{12}$ increases more and more rapidly with pressure thereby causing $c_{11}-c_{12}$ to vanish at approximately $47 \mathrm{GPa}$ (Fig. 1). This tetragonal shear instability has been shown to be associated with the transition from the tetragonal phase (stishovite) to the orthorhombic $\mathrm{CaCl}_{2}$ phase in $\mathrm{SiO}_{2}[$ Karki et al., 1997b; Cohen, 1992]. The transition is accompanied by a rapid decrease in the effective shear modulus, and a vanishing of its lower bound. In the $\mathrm{CaCl}_{2}$ phase, all elastic moduli increase monotonically with pressure except $c_{12}$ and $c_{23}$ which show an initial decrease followed by an increase with pressure. Finally all nine elastic stiffness coefficients of the columbite phase increase gradually with pressure. Both the stishovite-to- $\mathrm{CaCl}_{2}$ and $\mathrm{CaCl}_{2}$-to-columbite phase transitions in silica are clearly marked by discontinuities in the elastic moduli at pressures of 47 and $98 \mathrm{GPa}$.

\section{Elastic Wave Velocity and Anisotropy}

We calculate the single crystal elastic wave velocities of the stishovite, $\mathrm{CaCl}_{2}$ and columbite phases of silica along different propagation directions. Both the compressional (P) and shear (S) wave velocities are found to be strongly dependent on the propagation direction suggesting that silica is elastically highly anisotropic. We can define the azimuthal anisotropy for compressional $\left(A_{P}\right)$ and shear $\left(A_{S}\right)$ waves by following relations

$A_{P}=\frac{V_{P \max }-V_{P \min }}{V_{P a g g}} \times 100 ; \quad A_{S}=\frac{V_{S \max }-V_{S \min }}{V_{S_{a g g}}} \times 100$

where

$$
V_{\text {Pagg }}=\sqrt{\frac{K+\frac{4}{3} G}{\rho}} ; \quad V_{\text {Sagg }}=\sqrt{\frac{G}{\rho}}
$$

are the isotropic aggregate velocites, and $\rho$ is the density. The isotropic bulk $(K)$ and shear $(G)$ moduli are taken

Table 1. Calculated elastic moduli (in GPa) of three phases of silica.

\begin{tabular}{|c|c|c|c|c|c|c|c|c|c|c|c|}
\hline & $\mathbf{c 1 1}_{11}$ & $\mathbf{C}_{22}$ & C33 & C44 & Cs5 & $\mathbf{C}_{60}$ & $\mathbf{c}_{12}$ & $\mathbf{C}_{13}$ & $\mathbf{c}_{23}$ & $\mathbf{K}$ & G \\
\hline \multicolumn{12}{|c|}{ Stishovite at 0 GPa } \\
\hline Calc. & 462 & & 734 & 255 & & 324 & 210 & 195 & & 312 & 226 \\
\hline Lapv & 452 & & 807 & & & & 242 & 221 & & 314 & \\
\hline Expt. $^{2}$ & 453 & & $\begin{array}{l}776 \\
\mathrm{CaC}\end{array}$ & $\begin{array}{r}252 \\
2 \text { gtri }\end{array}$ & ctu & $\begin{array}{c}302 \\
\text { at } 50\end{array}$ & $\stackrel{211}{\mathbf{G P a}^{2}}$ & 203 & & 316 & 220 \\
\hline \multicolumn{11}{|c|}{ Columbite structure at $100 \mathrm{GPa}$} & 267 \\
\hline Calc. & 1031 & 1173 & 1217 & 367 & 393 & 454 & 528 & 474 & 422 & 696 & 371 \\
\hline
\end{tabular}

${ }^{1}$ Cohen, 1991, 1992

2 Weidner et al., 1982

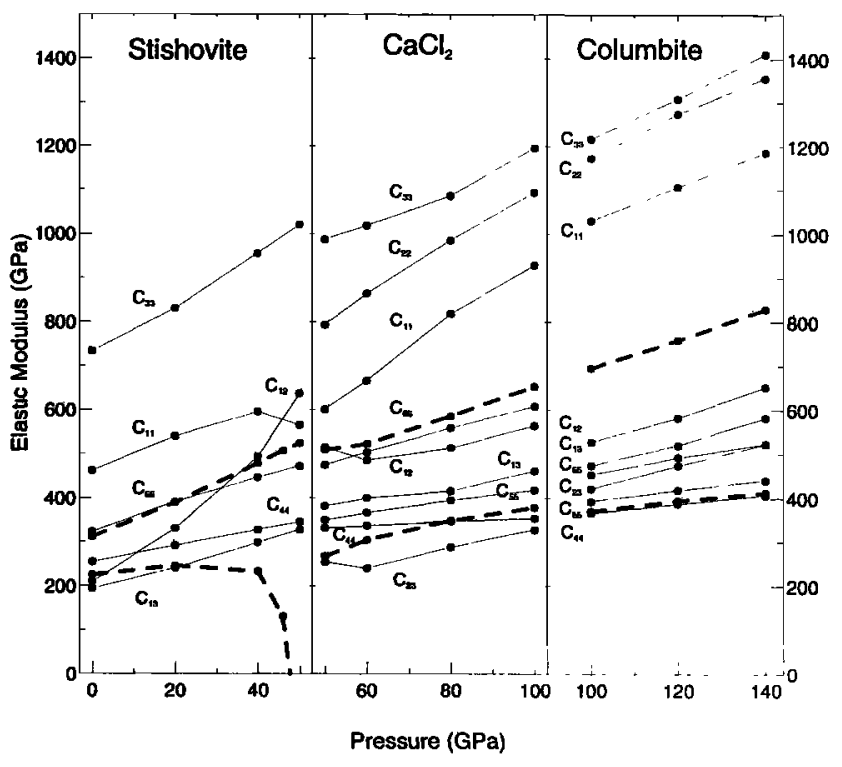

Figure 1. Pressure dependence of the elastic stiffness coefficients $\left(c_{i j}\right)$ of stishovite ( 0 to $\left.50 \mathrm{GPa}\right), \mathrm{CaCl}_{2}-(50$ to 100 $\mathrm{GPa}$ ) and columbite- (100 to $140 \mathrm{GPa}$ ) structure silica. The upper and lower dashed lines represent the isotropic bulk and shear moduli respectively.

from the Hashin-Shtrikman averaging scheme [Meister and Peselnick, 1966; Watt, 1979]. For the shear waves, the polarisation anisotropy can also be defined since there are two shear waves propagating with different velocities for a given propagation direction (i.e., S-wave birefringence). Silica is found to exhibit much stronger S-wave anisotropy than $\mathrm{P}$ wave anisotropy in all the three phases (Fig. 2). It becomes strongly anisotropic around the first transition point (47 GPa) whereas the anisotropy drops significantly at the second transition (98 GPa). The longitudinal and shear wave velocities vary by 23 and $45 \%$ respectively with direction at zero pressure whereas they vary by 32 and $175 \%$ respectively near the stishovite-to- $\mathrm{CaCl}_{2}$ transition point (at 46 $\mathrm{GPa}$ ). Polarization anisotropy increases by a factor of five over the same pressure range (from 31 to $148 \%$ ). Near this transition, the $\mathrm{CaCl}_{2}$ structure also shows large variations in velocities (P-azimuthal: $29 \%, \mathrm{~S}$-azimuthal: $76 \%$, and 56 $\%$ polarization at $50 \mathrm{GPa}$ ). Finally the columbite structure is much weaker in anisotropy than the other two structures.

Both $c_{11}$ and $c_{11}-c_{12}$ moduli soften strongly in the vicinity of the stishovite-to- $\mathrm{CaCl}_{2}$ phase transition point $\left(c_{11}-c_{12}\right.$ actually vanishes at $47 \mathrm{GPa}$ ), corresponding to very low-velocity $P$ - and $S$-waves in the [100] and [110] directions respectively and hence causing the anisotropy to be very strong around $47 \mathrm{GPa}$. Like the directions of the slowest wave propagation, the fastest propagation directions, [110] and [001] respectively for P- and S-waves, also remain unchanged at this transition. The maximum polarization anisotropy of S-waves occurs in the [110] direction at all pressures for both the stishovite and $\mathrm{CaCl}_{2}$ phases. In columbite phase, the fastest directions are [110] and [100] for $\mathrm{P}$ - and S-waves respectively whereas the slowest directions are [100] and [110] respectively, and the direction of the maximum velocity difference between polarizations for S-waves is $\left[1 \frac{1}{\sqrt{2}} \frac{1}{\sqrt{2}}\right]$. 


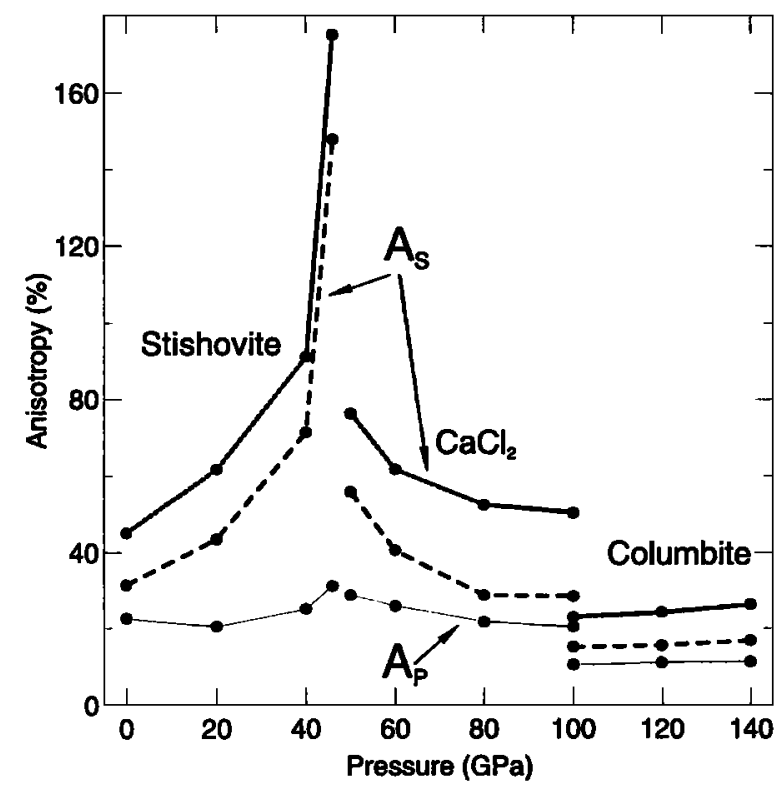

Figure 2. Pressure dependence of azimuthal anisotropy (solid line) in longitudinal wave velocity $\left(A_{P}\right)$, and azimuthal (dotted line) and polarisation (dashed line) anisotropy in shear $\left(A_{S}\right)$ wave velocity of silica.

\section{Geophysical Implications}

These results suggest that the elastic anisotropy of silica may be relevant in understanding the origin of observed seismic anisotropy. Free silica is not generally considered to be abundant in the mantle, however, its presence in small quantities cannot be ruled out at present. The calculated high pressure density of silica is slightly higher (by $3 \%$ ) whereas the $\mathrm{P}$ - and $\mathrm{S}$-wave velocities are significantly higher (by 12 and $18 \%$ respectively) than the corresponding seismic values of the lower mantle [Dziewonski and Anderson, 1981]. The anisotropy of silica is so strong, especially at pressures corresponding to the top of the lower mantle (148 $\%$ in polarization anisotropy near $1200 \mathrm{~km}$ depth), that even small amounts may in principle contribute significantly to observed anisotropy. In this context, it is worth emphasizing that models which attempt to explain seismic anisotropy must take into account the very strong pressure dependence of single crystal anisotropy in silica, and its pressure-induced phase transitions. The anisotropy of the columbite phase, the stable structure at pressures corresponding to the $\mathrm{D}^{\prime \prime}$ layer is very different from that of stishovite. The $\mathrm{D}^{\prime \prime}$ layer may contain more free silica than the rest of the lower mantle because of chemical reaction with the core [Knittle and Jeanloz, 1989]. Our predictions of the pressure dependence of elastic anisotropy bear in particular on recent studies which assumed that stishovite or the $\mathrm{CaCl}_{2}$ phase were stable under $\mathrm{D}^{\prime \prime}$ rather than the very different columbite structure [Kendall and Silver, 1996; Karato, 1997, ].

Silica undergoes two phase transitions within the pressure regime of the lower mantle which may provide an explanation of the origin of seismically reflective features near 920 $\mathrm{km}$ and $1200 \mathrm{~km}$ depth [Kawakatsu and Niu, 1994; Kingmo et al., 1995]. The stishovite to $\mathrm{CaCl}_{2}$ phase transition involves 20 and $60 \%$ discontinuous changes in $\mathrm{P}$ - and S-wave velocities respectively, compared to $1-2 \%$ changes associ- ated with the $\mathrm{CaCl}_{2}$ to columbite phase transition (Fig. 3). Although it has been recognized that the first transition in particular is associated with a large change in elastic properties, the ability of this transition to reflect signifcant amounts of seismic energy in the appropriate frequency range has not yet been examined.

To test the hypothesis that lower mantle reflectors are caused by the stishovite to $\mathrm{CaCl}_{2}$ phase transition, we have computed normal incidence frequency-dependent reflection coefficients, $R$, from this transition [Aki and Richards, 1980] (Fig. 4). The seismic velocity structure associated with the phase transition is unusual (Fig. 3). In the long wavelength limit, reflectivity is small, since the seismic velocities of the two phases far from the transition region itself are similar. However, in the immediate vicinity of the phase transition: 40-47 GPa, corresponding the depth range 1035$1180 \mathrm{~km}$ in the mantle, the elastic properties of stishovite change rapidly. The transition itself is sudden, occuring at a single pressure even in the multi-component mantle. This means that reflectivity at higher frequencies will be that of the step discontinuity associated with the transition.

Most travel time observations will see the phase transition as a step discontinuity. The reflection coefficient is approximately that of the step discontinuity (to within 50 \%) for frequencies greater than $10 \mathrm{mHz}$, lower than that of most travel time observations. The magnitude of $R$ for the step discontinuity is $8 \%$ for $\mathrm{P}$-waves and $29 \%$ for $\mathrm{S}$-waves. The uncertainties in these values are large because of inherent uncertainties in the elastic properties of aggregates: the Hashin-Shtrikman bounds become very broad in the vicinity of the transition (Fig. 3). The large reflectivities associated with the Hashing-Shtrikman averages mean that even small amounts of silica may in principle produce observable reflections. The estimated reflectivity of a $920 \mathrm{~km}$ feature in a multi-corridor stack of ScS reverberations is $0.5 \%$, requiring

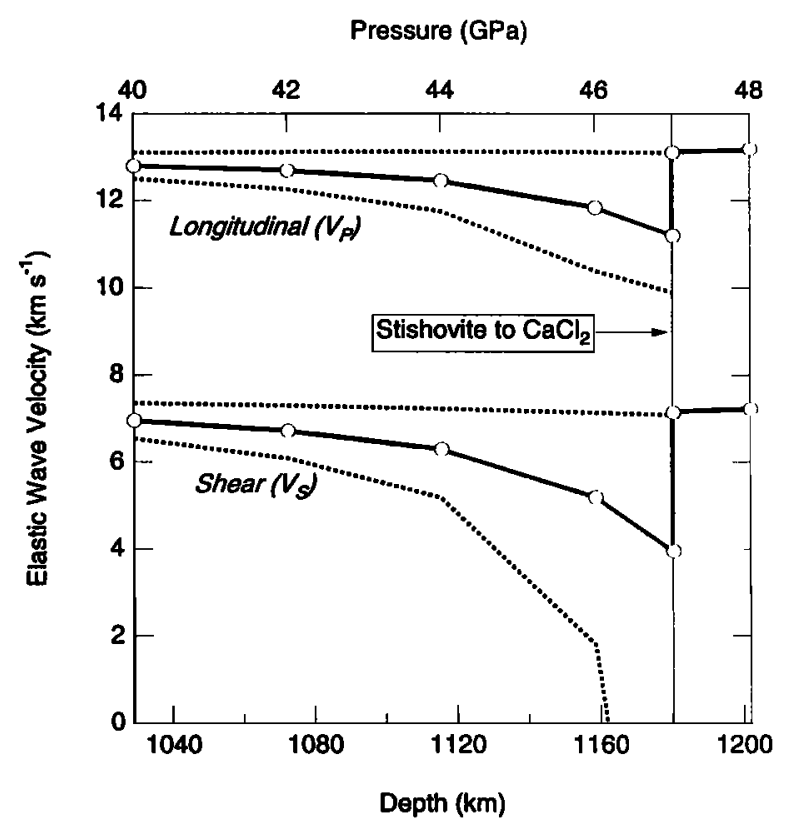

Figure 3. The velocity structure in the vicinity of the stishovite to $\mathrm{CaCl}_{2}$ phase transition (bold solid line with circles representing theoretical calculations) and the HashinShtrikman bounds (dashed lines). 


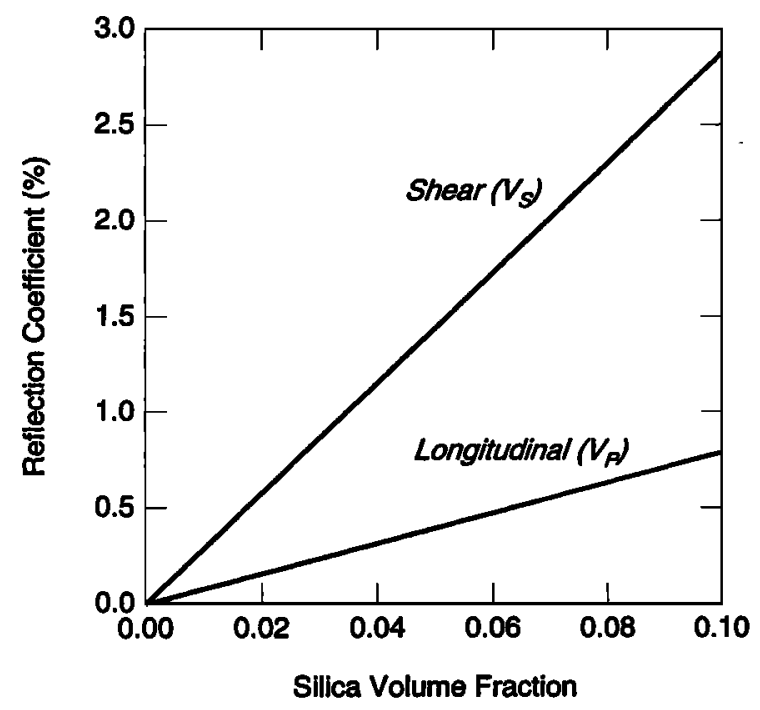

Figure 4. Normal incidence $\mathrm{P}$ - and $\mathrm{S}$-wave reflection coefficient of the stishovite to $\mathrm{CaCl}_{2}$ phase transition as a function of the volume fraction of silica in the lower mantle based on Hashin-Shtrikman average velocities.

2 volume percent silica to account for the transition, assuming Hashin-Shtrikman average velocities (Fig. 4). We note that while the transition occurs at a pressure corresponding to $1180 \mathrm{~km}$ depth in our athermal calculations, neither the sign, nor the magnitude of the Clapeyron slope of the transition is yet known, and it may occur at somewhat greater or shallower depths in the lower mantle.

\section{Conclusions}

Free silica is not generally considered to be present in the lower mantle. Small amounts, however, cannot be ruled out by present seismological observations and mineralogical data. We find that the elastic properties of silica are so unusual that even a few volume percent may be detectable with seismic methods. In particular, we find that stishovite at high pressure is one of the most anisotropic silicates known, with more than a factor of two polarization anisotropy. Moreover, this phase undergoes an unusual, second order phase transition at $47 \mathrm{GPa}$, which is driven by a shear instability. The transition results in a discontinuous change in shear wave velocity of approximately $60 \%$. This discontinuity is expected to reflect observable amounts of seismic energy if as little as two volume percent silica exists in the lower mantle. Silica may then provide an explanation of seismic observations of reflective features within the lower mantle. Silica is expected to adopt the columbite structure in the $\mathrm{D}^{\prime \prime}$ layer of the mantle, a phase with qualitatively different elastic anisotropy from either of the two lower pressure octahedral silica phases.

Acknowledgments. The authors thank EPSRC for computing facilities under Grant GRIK74067, M.C. Payne for the original CASTEP code, M.H. Lee for pseudopotentials and G.J. Ackland for useful discussions. B.B. Karki is supported by the University of Edinburgh under the Premier Scholarship. L. Stixrude acknowledges support from the National Science Foundation under grants EAR-9305060 and EAR-9628199. J. Crain acknowledges support from the Royal Society of Edinburgh.

\section{References}

Aki, K., and P. G. Richards, Quantitative Seismology, vol. I, W H. Freeman, New York, 1980.

Cohen, R. E., Bonding and elasticity of stishovite $\mathrm{SiO}_{2}$ at high pressure: Linearised augmented plane wave calculations, $A m$. Miner., 76, 733-742, 1991.

Cohen, R. E., First-principles predictions of elasticity and phase transitions in high pressure $\mathrm{SiO}_{2}$ and geophysical implications, High-pressure Research: Application to Earth and Planetary Sciences, edited by Y. Syono and M.H. Manghnani, 425-431, 1992.

Dziewonski, A. M., and D. L. Anderson, Preliminary reference earth model, Phys. Earth Planet. Int., 25, 297-356, 1981.

Francis, G. P., and M. C. Payne, Finite basis set corrections to total-energy pseudopotential calculations, J. Phys. C, 2, 43954404, 1990.

Karato, S., Seismic anisotropy in the deep mantle, boundary layers and the geometry of mantle convection, Pure. Appl. Geophys., (in press), 1997.

Karki, B. B., L. Stixrude, S. J. Clark, M. C. Warren, G. J. Ackland, and J. Crain, Structure and elasticity of $\mathrm{MgO}$ at high pressure, Am. Miner. 82, 51-60, 1997a.

Karki, B. B., M. C. Warren, L. Stixrude, G. J. Ackland, and J. Crain, $\mathrm{Ab}$ initio studies of high-pressure structural transformations in silica, Phys. Rev. B 55, 3465-3472, $1997 \mathrm{~b}$.

Note : The structure of silica which is misidentified as $\mathrm{Pnc}_{2}$ phase in the paper is identical to columbite $\left(\alpha-\mathrm{PbO}_{2}\right)$ structure with Pben space group; an erratum has been submitted to Phys. Rev. B.

Karki, B. B., L. Stixrude, S. J. Clark, M. C. Warren, G. J. Ackland, and J. Crain, Elastic properties of orthorhombic $\mathrm{MgSiO}_{3}$ perovskite at lower mantle pressures, Am. Miner., in press, 1997c.

Kawakatsu, H., and F. Niu, Seismic evidence for a 920-km discontinuity in the mantle, Nature, 371, 301-305, 1994.

Kendall, J.M., and P.G. Silver, Constraints from seismic anisotropy on the nature of the lowermost mantle, Nature, 381, 409-412, 1996.

Kingma, M. J., R.E. Cohen, R.J. Hemley, and H.K. Mao, Transformation of stishovite to a denser phase at lower mantle pressures, Nature, 374, 243-245, 1995.

Knittle, E., and R. Jeanloz, Simulation the core-mantle boundary: an experimental study of high-pressure reactions between silica and liquid iron, Geophys. Res. Lett., 16, 609612, 1989.

Kohn, W., and L. J. Sham, Self-consistent equations including exchange and correlation effects, Physical Review, 140, A1133A1138, 1965.

Meister, R. and L. Peselnick, Variational method of determining effective moduli of polycrystals with tetragonal symmetry, $J$. Appl. Phys., 37, 4121-4125, 1966.

Monkhurst, H. J., and J. D. Pack, Special points for Brillouinzone integrations, Phys. Rev. B Solid State, 13, 5188-5192, 1976

Watt, J.P., Hashin-Shtrikman bounds on the effective elastic moduli of polycrystals with orthorhombic symmetry, J. Appl. Phys., 50, 6290-6295, 1979.

Weidner, D. J., J. D. Bass, A. E. Ringwood, and W. Sinclair, The single-crystal elastic moduli of stishovite, J. Geophys. Res., 87, B4740-B4746, 1982.

Wentzcovitch, R. M., N. L. Ross, and G. D. Price, Ab initio study of $\mathrm{MgSiO}_{3}$ and $\mathrm{CaSiO}_{3}$ perovskites at lower mantle pressures, Phys. Earth Planet. Int., 90, 101-112, 1995.

Bijaya B. Karki and Jason Crain, Department of Physics and Astronomy, The University of Edinburgh, Edinburgh, EH9 3JZ, Scotland, U.K.

Lars Stixrude, School of Earth and Atmospheric Sciences, Georgia Institute of Technology, Atlanta, GA 30332-0340, U.S.A.

(Received May 21, 1997; accepted October 1, 1997.) 